\title{
Effects of pelargonidin-3-O-glucoside and its metabolites on lipopolysaccharide- stimulated cytokine production by THP-1 monocytes and macrophages
}

Article

Accepted Version

Creative Commons: Attribution-Noncommercial-No Derivative Works 4.0

Amini, A. M., Spencer, J. P. E. and Yaqoob, P. (2018) Effects of pelargonidin-3-O-glucoside and its metabolites on lipopolysaccharide-stimulated cytokine production by THP-1 monocytes and macrophages. Cytokine, 103. pp. 29-33. ISSN 1096-0023 doi: https://doi.org/10.1016/j.cyto.2017.12.031 Available at https://centaur.reading.ac.uk/75012/

It is advisable to refer to the publisher's version if you intend to cite from the work. See Guidance on citing.

To link to this article DOI: http://dx.doi.org/10.1016/j.cyto.2017.12.031

Publisher: Elsevier

All outputs in CentAUR are protected by Intellectual Property Rights law, including copyright law. Copyright and IPR is retained by the creators or other copyright holders. Terms and conditions for use of this material are defined in the End User Agreement. 


\section{CentAUR}

Central Archive at the University of Reading

Reading's research outputs online 
1 Effects of pelargonidin-3-O-glucoside and its metabolites on lipopolysaccharide-

2 stimulated cytokine production by THP-1 monocytes and macrophages

4 Anna M. Amini ${ }^{a}$, Jeremy PE. Spencer ${ }^{\mathrm{a}}$, Parveen Yaqoob $^{\mathrm{a}}$

6 a Department of Food and Nutritional Sciences, University of Reading, Whiteknights PO Box $7 \quad$ 226, Reading RG6 6AP, UK

9 Corresponding author: Parveen Yaqoob, Telephone: +44 (0) 118378 8720, E-mail:

10 p.yaqoob@reading.ac.uk

12 Anna M. Amini’s E-mail: amini.anna@gmail.com

13 Jeremy PE. Spencer's E-mail: j.p.e.spencer@ reading.ac.uk

15 Abbreviations: 4-HBA, 4-hydroxybenzoic acid; CVD, cardiovascular disease; FCS, fetal

16 calf serum; IL, interleukin; LPS, lipopolysaccharide; PCA, protocatechuic acid; Pg-3-glc,

17 pelargonidin-3-O-glucoside; PGA, phloroglucinaldehyde; PMA, phorbol-12-myristate-13-

18 acetate; TNF- $\alpha$, tumor necrosis factor- $\alpha$; 


\section{Abstract}

20 Epidemiological evidence suggests cardioprotective effects of anthocyanin consumption. This

21 study examined the predominant strawberry anthocyanin, pelargonidin-3-O-glucoside (Pg-3-

22 glc), and three of its plasma metabolites (protocatechuic acid [PCA], 4-hydroxybenzoic acid,

23 and phloroglucinaldehyde [PGA]) for effects on the production of selected cytokines by

24 lipopolysaccharide-stimulated THP-1 monocytes and macrophages. Concentrations of tumor

25 necrosis factor- $\alpha$ (TNF- $\alpha$ ), interleukin (IL)-1 $\beta$, IL-6, IL-8 and IL-10 were determined using a

26 cytometric bead array kit. PCA at $0.31,1.25$ and $20 \mu \mathrm{M}$ and PGA at 5 and $20 \mu \mathrm{M}$ decreased

27 the concentration of IL- 6 in the monocyte cultures, but there were no effects on TNF- $\alpha$, IL-1 $\beta$,

28 IL-8 and IL-10 and there were no effects of the other compounds. In the macrophage cultures,

29 PGA at $20 \mu \mathrm{M}$ decreased the concentrations of IL-6 and IL-10, but there was no effect on

30 TNF- $\alpha$, IL-1 $\beta$ and IL-8 and there were no effects of the other compounds. In conclusion,

31 while the effects of PGA were only observed at the higher, supraphysiological concentration

32 and are thus considered of limited physiological relevance overall, the anti-inflammatory

33 properties of PCA were observed at both the lower, physiologically relevant, and the higher

34 concentrations; however, effects were modest and limited to IL-6 and monocytes. These

35 preliminary data suggest potential for physiologically attainable PCA concentrations to

36 modulate IL-6 production by monocytes.

38 Keywords: Anthocyanin, Cytokine, Inflammation, Pelargonidin-3-O-glucoside, Strawberry 


\section{$39 \quad 2.1$ Introduction}

40 Epidemiological evidence links anthocyanin consumption with lower risk of cardiovascular

41 disease (CVD) [1] and CVD risk factors [2]. The mechanisms are not yet fully elucidated, but

42 evidence suggests modulation of vascular function, platelet aggregation and inflammation [3-

43 9]. TNF- $\alpha$, IL-1 $\beta$, IL-6, IL-8 and IL-10 are inflammatory cytokines that play a critical role in

44 atherosclerosis, the underlying cause of most CVDs [10].

45 Strawberries are particularly rich in anthocyanins, predominantly pelargonidin-3-O-glucoside

46 (Pg-3-glc). Glucuronidated pelargonidin has been reported as the predominant metabolite in

47 three pharmacokinetic studies [11-13], but there is ambiguity regarding the position of

48 glucuronidation and glucuronidated pelargonidin compounds are currently commercially

49 unavailable and hence cannot be tested in cellular models. 4-hydroxybenzoic acid (4-HBA)

50 and protocatechuic acid (PCA) have also been reported in plasma following strawberry

51 consumption in low micromolar concentrations $(0.1-2 \mu \mathrm{M})$ [13-15]. In addition, it is likely

52 that phloroglucinaldehyde (PGA) might appear in plasma following strawberry consumption,

53 as it is a A-ring degradant, reported in plasma upon anthocyanin consumption in low to high

54 nanomolar concentrations $(20-600 \mathrm{nM})[16,17]$. However, most studies exploring the effect

55 of anthocyanins to modulate cytokine secretion used unmetabolized parent anthocyanins,

56 often at supraphysiologically high doses [3, 4]. In addition, although macrophages are an

57 important source of inflammatory cytokines [18], there are no studies exploring the potential

58 modulation of cytokine production by anthocyanins or their metabolites by human-derived

59 macrophages, or studies comparing the effects in human-derived monocytes versus

60 macrophages. Thus, the aim of this study was to examine the parent anthocyanin Pg-3-glc and

61 three physiologically relevant plasma metabolites for effects on the production of selected 
62 pro- and anti-inflammatory cytokines (tumor necrosis factor- $\alpha$ [TNF- $\alpha$ ], interleukin [IL]-1 $\beta$, 63 IL-6, IL-8 and IL-10) in lipopolysaccharide (LPS)-induced THP-1 cells. 


\section{$64 \quad 2.2$ Materials and methods}

\section{$65 \quad$ 2.2.1 Chemicals and reagents}

66 Pg-3-glc was purchased from Extrasynthese (Genay, France). PCA (3,4-dihydroxybenzoic

67 acid), 4-HBA, PGA (2,4,6-trihydroxybenzaldehyde), LPS from Escherichia coli, phorbol 12-

68 myristate 13-acetate (PMA), methanol and formic acid were purchased from Sigma-Aldrich

69 (Dorset, United Kingdom). RPMI 1640 culture medium, fetal calf serum (FCS), penicillin

70 and streptomycin were purchased from Lonza (Basel, Switzerland). The cytometric bead array kit to analyze cytokine concentrations was purchased from BD Biosciences (Oxford,

72 United Kingdom).

\section{$73 \quad$ 2.2.2 Preparation and culture of THP-1 cells}

74 THP-1 cells (human monocytic leukemia, ECACC 88081201) were cultured in RPMI 1640

75 culture medium supplemented with $100 \mathrm{UI} / \mathrm{mL}$ streptomycin, $100 \mu \mathrm{g} / \mathrm{mL}$ penicillin and $10 \%$

76 (v/v) $\mathrm{FCS}$ at $37^{\circ} \mathrm{C}$ in a humidified atmosphere of $5 \% \mathrm{CO}_{2}$ and kept at a density of 2-

$779 \times 10^{5}$ cells $/ \mathrm{mL}$. For the experiments, cells were seeded in 24 well plates at a density of

$781 \times 10^{6}$ cells $/ \mathrm{mL}$. For differentiation into macrophages, cells were exposed to PMA at a final

79 concentration of $0.1 \mu \mathrm{M}$ for $72 \mathrm{~h}$ [19]. Polyphenols were added to provide final

80 concentrations of $0.08,0.31,1.25,5$ and $20 \mu \mathrm{M}$. Polyphenols were added in $20 \mu \mathrm{L}$ of $10.98 \%$

81 methanol and $0.22 \%$ formic acid to produce a final concentration of $0.22 \%$ methanol and

$820.004 \%$ formic acid in culture. The final culture volume was $1 \mathrm{~mL}$. LPS $(20 \mu \mathrm{L} ; 1 \mu \mathrm{g} / \mathrm{mL}$

83 final concentration) was added to stimulate cytokine production. 
84 After $24 \mathrm{~h}$ incubation at $37^{\circ} \mathrm{C}$ in a humidified atmosphere of $5 \% \mathrm{CO}_{2}$, plates were 85 centrifuged at $260 \times \mathrm{g}$ for $5 \mathrm{~min}$ and culture supernatants were collected and stored in aliquots 86 at $-20^{\circ} \mathrm{C}$ until analysis.

\section{$87 \quad$ 2.2.3 Measurement of cytokine concentrations}

88 Concentrations of TNF- $\alpha$, IL- $1 \beta$, IL- 6 , IL- 8 and IL-10 in the culture supernatants were 89 measured using a cytometric bead array kit from BD Biosciences (Oxford, United Kingdom)

90 according to the manufacturer's instructions. Data were acquired on a BD FACS Canto ${ }^{\mathrm{TM}}$ II

91 flow cytometer and analyzed using the BD FCAP Array v3 software. Limits of detection of 92 the cytokine assays were $0.13 \mathrm{pg} / \mathrm{mL}$ (IL-10), $1.2 \mathrm{pg} / \mathrm{mL}$ (TNF- $\alpha$ and IL-8), $1.6 \mathrm{pg} / \mathrm{mL}$ (IL-6) 93 and $2.3 \mathrm{pg} / \mathrm{mL}$ (IL-1 $\beta$ ).

\section{$94 \quad$ 2.2.4 Cytotoxicity}

95 To determine whether test compounds had any cytotoxic effects, cell viability of THP-1 96 monocytes and macrophages was assessed using Trypan blue staining.

\section{$97 \quad$ 2.2.5 Statistical analyses}

98 Results are expressed as percentage of cytokine production versus control (no polyphenols)

99 and shown as means with their standard deviations (SD). One-way ANOVA was performed

100 to determine whether test compounds affected the cytokine production, followed by Dunnett

101 as post hoc analysis versus control group. Statistical analysis was performed using SPSS 21

102 (IBM Corporation, New York, USA) and a lowered $P<0.01$ was considered significant to 103 account for multiple comparisons. 


\subsubsection{Effects of Pg-3-glc, PCA, 4-HBA and PGA on viability of THP-1 cells}

107 There were no cytotoxic effects of the studied compounds on THP-1 monocytes or 108 macrophages at any of the tested doses (data not shown).

\subsubsection{Effects of Pg-3-glc, PCA, 4-HBA and PGA on cytokine production by THP-1 monocytes}

111 Stimulation with LPS increased IL-1 $\beta$ production 135 -fold, TNF- $\alpha$ production 105 -fold, IL-6

112 production 470-fold, IL-8 production 640-fold and IL-10 production 5-fold. PCA

113 significantly reduced IL-6 production at $0.31,1.25$ and $20 \mu \mathrm{M}$ compared to the control

114 cultures (all $P<0.01$, Table 1). PGA also significantly inhibited IL-6 production and while the

115 effects were only significant at 5 and $20 \mu \mathrm{M}(P<0.01$ and $P<0.001$ respectively, Table 1$)$,

116 they were slightly more potent (production was lowered by $25-35 \%$ compared to $20 \%$ for

117 PCA) and appeared to be concentration-dependent. There was no significant effect of any

118 tested compound, at concentrations up to $20 \mu \mathrm{M}$, on the production of IL- $1 \beta$, TNF- $\alpha$, IL- 8 or

119 IL-10 by THP-1 monocytes (Table 1).

\subsubsection{Effects of Pg-3-glc, PCA, 4-HBA and PGA on cytokine production by THP-1 macrophages}

122 Stimulation with LPS increased IL-1 $\beta$ production 10 -fold, TNF- $\alpha$ production 45 -fold, IL-6

123 production 220-fold, IL-8 production 80-fold and IL-10 production 5-fold. PGA was the only

124 compound that induced significant changes in cytokine production. PGA at the highest dose 
125 tested $(20 \mu \mathrm{M})$ significantly lowered IL-6 production $(P<0.001$, Table 2$)$, similar to the 126 monocyte results and with a similar magnitude of effect. Furthermore, it also significantly 127 decreased IL-10 production $(20 \mu \mathrm{M} ; P<0.01$; Table 2$)$, an effect not observed in THP-1 128 monocytes. There was no significant effect of any of the tested compounds, at concentrations 129 up to $20 \mu \mathrm{M}$, on the production of IL-1 $\beta$, TNF- $\alpha$ or IL- 8 by THP-1 macrophages (Table 2). 


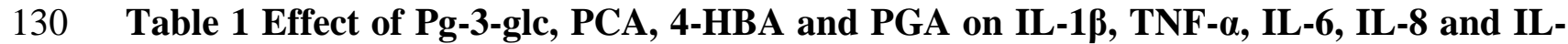
13110 production by THP-1 monocytes

\begin{tabular}{|c|c|c|c|c|c|}
\hline \multirow[b]{3}{*}{ Pg-3-glc } & \multicolumn{5}{|c|}{ Cytokine production (\% of control) } \\
\hline & \multirow[t]{2}{*}{ IL-1及 } & \multirow[t]{2}{*}{ TNF- $\alpha$} & IL-6 & \multirow[t]{2}{*}{ IL-8 } & \multirow[t]{2}{*}{ IL-10 } \\
\hline & & & & & \\
\hline $0 \mu \mathrm{M}$ & 100 & 100 & 100 & 100 & 100 \\
\hline $0.08 \mu \mathrm{M}$ & $91.8 \pm 18.9$ & $114.2 \pm 20.5$ & $85.6 \pm 17.5$ & $108.6 \pm 15.6$ & $113.4 \pm 12.4$ \\
\hline $0.31 \mu \mathrm{M}$ & $92.7 \pm 10.7$ & $110.8 \pm 20.3$ & $78.2 \pm 13.2$ & $102.3 \pm 19.6$ & $104.0 \pm 8.9$ \\
\hline $1.25 \mu \mathrm{M}$ & $93.2 \pm 8.4$ & $112.9 \pm 20.1$ & $78.4 \pm 11.8$ & $96.1 \pm 13.0$ & $102.9 \pm 10.4$ \\
\hline $5.00 \mu \mathrm{M}$ & $92.3 \pm 9.6$ & $106.4 \pm 19.4$ & $84.5 \pm 10.4$ & $101.9 \pm 12.1$ & $102.5 \pm 8.0$ \\
\hline $20.00 \mu \mathrm{M}$ & $96.4 \pm 13.9$ & $101.1 \pm 20.0$ & $88.5 \pm 18.6$ & $103.9 \pm 19.4$ & $98.0 \pm 4.9$ \\
\hline \multicolumn{6}{|l|}{ PCA } \\
\hline $0 \mu \mathrm{M}$ & 100 & 100 & 100 & 100 & 100 \\
\hline $0.08 \mu \mathrm{M}$ & $94.5 \pm 7.8$ & $103.5 \pm 18.9$ & $87.3 \pm 7.0$ & $110.3 \pm 13.0$ & $107.0 \pm 6.9$ \\
\hline $0.31 \mu \mathrm{M}$ & $97.7 \pm 7.5$ & $93.4 \pm 15.3$ & $77.8 \pm 9.7 * *$ & $98.2 \pm 7.1$ & $104.6 \pm 5.9$ \\
\hline $1.25 \mu \mathrm{M}$ & $104.9 \pm 5.2$ & $92.9 \pm 10.7$ & $77.2 \pm 10.2 * *$ & $106.0 \pm 9.0$ & $105.4 \pm 7.0$ \\
\hline $5.00 \mu \mathrm{M}$ & $95.2 \pm 12.8$ & $85.7 \pm 7.9$ & $88.3 \pm 15.9$ & $100.5 \pm 14.9$ & $99.0 \pm 9.5$ \\
\hline $20.00 \mu \mathrm{M}$ & $89.3 \pm 19.3$ & $85.9 \pm 11.0$ & $79.8 \pm 14.1 * *$ & $102.4 \pm 10.1$ & $93.6 \pm 7.6$ \\
\hline \multicolumn{6}{|l|}{ 4-HBA } \\
\hline $0 \mu \mathrm{M}$ & 100 & 100 & 100 & 100 & 100 \\
\hline $0.08 \mu \mathrm{M}$ & $83.4 \pm 7.6$ & $104.2 \pm 21.2$ & $99.1 \pm 14.5$ & $94.0 \pm 18.2$ & $99.9 \pm 8.6$ \\
\hline $0.31 \mu \mathrm{M}$ & $97.7 \pm 10.4$ & $109.3 \pm 22.5$ & $90.9 \pm 5.1$ & $91.3 \pm 15.7$ & $104.8 \pm 8.9$ \\
\hline $1.25 \mu \mathrm{M}$ & $97.3 \pm 15.3$ & $110.4 \pm 26.4$ & $91.9 \pm 3.6$ & $90.9 \pm 16.5$ & $101.8 \pm 6.9$ \\
\hline $5.00 \mu \mathrm{M}$ & $100.2 \pm 8.6$ & $107.2 \pm 19.5$ & $85.1 \pm 21.6$ & $93.5 \pm 17.8$ & $103.7 \pm 8.0$ \\
\hline $20.00 \mu \mathrm{M}$ & $105.7 \pm 13.0$ & $114.2 \pm 16.5$ & $93.4 \pm 25.0$ & $90.2 \pm 22.2$ & $102.3 \pm 7.1$ \\
\hline \multicolumn{6}{|l|}{ PGA } \\
\hline $0 \mu \mathrm{M}$ & 100 & 100 & 100 & 100 & 100 \\
\hline $0.08 \mu \mathrm{M}$ & $97.2 \pm 11.6$ & $111.4 \pm 21.3$ & $90.0 \pm 16.2$ & $103.2 \pm 13.7$ & $110.6 \pm 13.1$ \\
\hline $0.31 \mu \mathrm{M}$ & $102.1 \pm 7.4$ & $107.5 \pm 21.4$ & $91.9 \pm 17.2$ & $96.8 \pm 21.1$ & $104.6 \pm 11.0$ \\
\hline $1.25 \mu \mathrm{M}$ & $106.5 \pm 11.8$ & $112.9 \pm 18.9$ & $79.7 \pm 10.6$ & $97.0 \pm 13.8$ & $101.2 \pm 9.0$ \\
\hline $5.00 \mu \mathrm{M}$ & $110.4 \pm 12.3$ & $117.0 \pm 22.1$ & $73.6 \pm 6.9 * *$ & $95.2 \pm 20.6$ & $97.1 \pm 9.2$ \\
\hline $20.00 \mu \mathrm{M}$ & $106.2 \pm 12.6$ & $111.6 \pm 21.7$ & $65.9 \pm 17.6 * * *$ & $94.6 \pm 19.3$ & $95.6 \pm 6.1$ \\
\hline
\end{tabular}

132 THP-1 monocytes $\left(1 \times 10^{6}\right.$ cells/mL) were treated with Pg-3-glc, PCA, 4-HBA, PGA or vehicle control at 133 concentrations of $0-20 \mu \mathrm{M}$, prior to lipopolysaccharide stimulation $(1 \mu \mathrm{g} / \mathrm{mL})$ and incubated for $24 \mathrm{~h}$ at $37^{\circ} \mathrm{C}$. 134 Cytokine concentrations in the culture supernatants were measured using a cytometric bead array kit. Results are 135 expressed as percentage of cytokine concentration vs. control (no polyphenols). Data are represented as the 136 mean \pm SD of six independent experiments. Data were analyzed by one-way ANOVA and Dunnett post hoc 137 analysis, where applicable, and a lowered $\mathrm{P}<0.01$ was considered significant to account for multiple 138 comparisons. Statistically significant differences are denoted as $* * P<0.01$ vs. control; $* * * P<0.001$ vs. control. 139 4-HBA, 4-hydroxybenzoic acid; IL, interleukin; PCA, protocatechuic acid; Pg-3-glc, pelargonidin-3-O140 glucoside; PGA, phloroglucinaldehyde; TNF- $\alpha$, tumor necrosis factor- $\alpha$. 


\begin{tabular}{|c|c|c|c|c|c|}
\hline \multirow{3}{*}{ Pg-3-glc } & \multicolumn{5}{|c|}{ Cytokine production ( $\%$ of control) } \\
\hline & \multirow[t]{2}{*}{ IL-1及 } & \multirow[t]{2}{*}{ TNF- $\alpha$} & \multirow[t]{2}{*}{ IL-6 } & \multirow[t]{2}{*}{ IL-8 } & \multirow[t]{2}{*}{ IL-10 } \\
\hline & & & & & \\
\hline $0 \mu \mathrm{M}$ & 100 & 100 & 100 & 100 & 100 \\
\hline $0.08 \mu \mathrm{M}$ & $113.2 \pm 18.8$ & $117.3 \pm 31.0$ & $113.5 \pm 24.7$ & $111.4 \pm 12.8$ & $100.9 \pm 17.4$ \\
\hline $0.31 \mu \mathrm{M}$ & $111.6 \pm 15.1$ & $115.8 \pm 22.2$ & $108.0 \pm 21.6$ & $106.5 \pm 12.2$ & $87.3 \pm 15.1$ \\
\hline $1.25 \mu \mathrm{M}$ & $108.9 \pm 12.3$ & $112.4 \pm 16.0$ & $101.4 \pm 24.1$ & $105.8 \pm 16.9$ & $88.0 \pm 16.8$ \\
\hline $5.00 \mu \mathrm{M}$ & $107.8 \pm 16.7$ & $110.5 \pm 24.5$ & $101.6 \pm 20.1$ & $103.4 \pm 16.1$ & $80.1 \pm 13.5$ \\
\hline $20.00 \mu \mathrm{M}$ & $99.2 \pm 14.7$ & $106.0 \pm 18.1$ & $107.7 \pm 19.8$ & $102.9 \pm 11.8$ & $84.3 \pm 16.0$ \\
\hline \multicolumn{6}{|l|}{ PCA } \\
\hline $0 \mu \mathrm{M}$ & 100 & 100 & 100 & 100 & 100 \\
\hline $0.08 \mu \mathrm{M}$ & $102.7 \pm 9.3$ & $117.2 \pm 23.2$ & $116.2 \pm 25.2$ & $106.5 \pm 16.1$ & $100.8 \pm 12.0$ \\
\hline $0.31 \mu \mathrm{M}$ & $103.8 \pm 10.0$ & $101.0 \pm 18.6$ & $111.7 \pm 22.9$ & $104.4 \pm 14.1$ & $85.0 \pm 24.9$ \\
\hline $1.25 \mu \mathrm{M}$ & $100.8 \pm 6.8$ & $96.5 \pm 26.0$ & $101.8 \pm 23.6$ & $101.7 \pm 12.5$ & $84.1 \pm 22.2$ \\
\hline $5.00 \mu \mathrm{M}$ & $100.9 \pm 10.8$ & $101.7 \pm 17.5$ & $107.2 \pm 23.9$ & $98.7 \pm 12.0$ & $83.2 \pm 19.6$ \\
\hline $20.00 \mu \mathrm{M}$ & $99.1 \pm 9.9$ & $100.6 \pm 22.9$ & $88.6 \pm 19.4$ & $103.7 \pm 12.0$ & $77.8 \pm 27.2$ \\
\hline \multicolumn{6}{|l|}{ 4-HBA } \\
\hline $0 \mu \mathrm{M}$ & 100 & 100 & 100 & 100 & 100 \\
\hline $0.08 \mu \mathrm{M}$ & $108.7 \pm 17.9$ & $115.3 \pm 28.7$ & $107.9 \pm 21.4$ & $111.3 \pm 13.6$ & $106.2 \pm 13.5$ \\
\hline $0.31 \mu \mathrm{M}$ & $107.4 \pm 17.1$ & $112.2 \pm 27.7$ & $104.2 \pm 17.0$ & $103.4 \pm 16.6$ & $93.1 \pm 15.0$ \\
\hline $1.25 \mu \mathrm{M}$ & $110.2 \pm 18.2$ & $109.6 \pm 29.3$ & $101.1 \pm 14.4$ & $102.6 \pm 19.5$ & $90.8 \pm 19.0$ \\
\hline $5.00 \mu \mathrm{M}$ & $109.9 \pm 16.8$ & $97.8 \pm 27.3$ & $97.4 \pm 27.4$ & $103.0 \pm 15.2$ & $88.5 \pm 16.5$ \\
\hline $20.00 \mu \mathrm{M}$ & $112.2 \pm 18.4$ & $109.7 \pm 27.9$ & $94.7 \pm 25.8$ & $95.2 \pm 16.1$ & $94.8 \pm 11.6$ \\
\hline \multicolumn{6}{|l|}{ PGA } \\
\hline $0 \mu \mathrm{M}$ & 100 & 100 & 100 & 100 & 100 \\
\hline $0.08 \mu \mathrm{M}$ & $106.6 \pm 7.4$ & $113.9 \pm 21.7$ & $109.5 \pm 17.6$ & $110.9 \pm 16.4$ & $110.3 \pm 21.2$ \\
\hline $0.31 \mu \mathrm{M}$ & $111.4 \pm 11.9$ & $107.6 \pm 18.0$ & $95.7 \pm 18.6$ & $104.8 \pm 11.9$ & $77.6 \pm 15.3$ \\
\hline $1.25 \mu \mathrm{M}$ & $112.3 \pm 8.7$ & $100.5 \pm 18.7$ & $79.7 \pm 16.0$ & $103.1 \pm 21.2$ & $72.1 \pm 16.0$ \\
\hline $5.00 \mu \mathrm{M}$ & $110.9 \pm 16.3$ & $98.5 \pm 21.4$ & $71.8 \pm 19.1$ & $106.3 \pm 12.3$ & $70.9 \pm 19.1$ \\
\hline $20.00 \mu \mathrm{M}$ & $113.7 \pm 17.9$ & $110.5 \pm 23.7$ & $58.8 \pm 25.6 * * *$ & $115.6 \pm 23.2$ & $65.4 \pm 26.2 * *$ \\
\hline
\end{tabular}

143 THP-1 monocytes $\left(1 \times 10^{6}\right.$ cells $\left./ \mathrm{mL}\right)$ were converted to macrophages through exposure to $0.1 \mu \mathrm{M}$ phorbol-12144 myristate-13-acetate for $72 \mathrm{~h}$. THP-1 macrophages were treated with Pg-3-glc, PCA, 4-HBA, PGA or vehicle control at concentrations of $0-20 \mu \mathrm{M}$, prior to lipopolysaccharide stimulation $(1 \mu \mathrm{g} / \mathrm{mL})$ and incubated for $24 \mathrm{~h}$ at $37{ }^{\circ} \mathrm{C}$. Cytokine concentrations in the culture supernatants were measured using a cytometric bead array kit. Results are expressed as percentage of cytokine concentration vs. control (no polyphenols). Data are represented as the mean \pm SD of six independent experiments. Data were analyzed by one-way ANOVA and Dunnett post hoc analysis, where applicable, and a lowered $P<0.01$ was considered significant to account for multiple comparisons. Statistically significant differences are denoted as $* * P<0.01$ vs. control; $* * * P<0.001$ vs. control. 


\subsection{Discussion}

154 This study aimed to compare the effects of Pg-3-glc and its plasma metabolites PCA, 4-HBA

155 and PGA on cytokine secretion by LPS-stimulated THP-1 cells. There were modest anti-

156 inflammatory effects of some of the tested compounds in THP-1 monocytes, with PCA and

157 PGA inhibiting IL-6 production, but there were no effects on TNF- $\alpha$, IL-1 $\beta$, IL-8 and IL-10

158 and there were no effects of the other compounds. The effects in macrophages were slightly

159 different. Whilst PGA inhibited IL-6 production by THP-1 derived macrophages, it also

160 inhibited IL-10 production, which was not observed in THP-1 monocytes.

161 IL-6 is generally classified as a pro-inflammatory cytokine and the inhibition of LPS-

162 stimulated IL-6 secretion by PCA and PGA in the monocyte cultures and PGA in the

163 macrophage cultures is a novel observation. Importantly, the inhibitory effect of PCA was

164 observed at physiologically attainable [14, 15, 17] low micromolar concentrations. Taken

165 together with previous investigations, the current results contribute to the suggestion that

166 PCA acts on multiple cell types involved in IL-6 secretion during atherosclerosis, including

167 endothelial [7] and dendritic cells [4], although the latter experiment applied a

168 supraphysiological PCA dose of $25 \mu \mathrm{M}$. It is important to note in this context that the

169 presence of PCA in plasma has also been reported following ingestion of other anthocyanins

$170[42,53,54]$, the flavonol quercetin [55] and it is also naturally present in several other dietary

171 sources, such as chicory, olives, raspberries, dates and onions [2, 56, 57], but it is currently

172 not known what proportion in plasma comes from these sources and what proportion

173 indirectly as a metabolite. Whilst the bioactivity of PGA on IL-6 was a novel observation, the

174 effect was only significant at the higher 5 and $20 \mu \mathrm{M}$ concentrations. As levels in circulation

175 remain below micromolar concentration (even after anthocyanin consumption), this finding is

176 likely of limited physiological relevance [16, 17]. 
177 IL-10 is generally classified as an anti-inflammatory cytokine. PGA decreased the production

178 of IL-10 in THP-1 macrophages, suggesting a pro-inflammatory tendency and this seems to

179 contrast its IL-6-reducing (i.e. anti-inflammatory) effect. However, the IL-10 decreasing

180 effect was observed only at high concentrations that are unlikely to be achieved in vivo. To

181 our knowledge, no other studies have investigated the effect of PGA on IL-10 production by

182 monocytes or macrophages. There is evidence to suggest that the inhibition of IL-6 and IL-10

183 production could be linked in a way that inhibition of IL-6 production could indirectly inhibit

184 IL-10 production [20]. There were no effects of any of the other tested compounds on IL-10

185 levels, which is in line with experiments on the effect of PCA in human monocyte-derived

186 dendritic cells [4] and Pg-3-glc, PCA and 4-HBA in THP-1 monocytes [6]. Beneficial effects

187 on IL-10 production were observed in human leukocytes with tea-derived polyphenols [21],

188 suggesting differential bioactivity of different polyphenol classes, although effects were only

189 observed at the higher, supraphysiological polyphenol doses (10 and $20 \mu \mathrm{M})$.

190 4-HBA had no effect on the secretion of any of the cytokines. Consistent with this, 4-HBA at

$191 \quad 0.1-10 \mu \mathrm{M}$ was previously reported to have no effect on TNF- $\alpha$ secretion by LPS-stimulated

192 THP-1 monocytes. Interestingly, however, this study is indicative of additive/synergistic

193 effects between polyphenols as an inhibitory effect on TNF- $\alpha$ was reported when 4-HBA was

194 coincubated with PCA or PCA plus vanillic acid, none of which was bioactive in isolation [6].

195 Furthermore, the reported IL-1 $\beta$-reducing effect of 4-HBA is in contrast to the present study,

196 despite both studies employing similar concentrations of 4-HBA, the same cell line and

197 similar culture conditions, but the current experiment conducted no preincubation with test

198 compounds. In peripheral blood mononuclear cells, $1 \mu \mathrm{M} 4$-HBA had no effect on IL-1 $\beta$ or

199 IL-6 levels in accordance with the present data, but a modest $10 \%$ increase in TNF- $\alpha$ levels

200 was observed, which could be related to the differences in cell type and culture conditions. 
201 The discrepant findings regarding the effect of 4-HBA on IL-1 $\beta$ and TNF- $\alpha$ merit further 202 investigation.

203 In this study only PCA and PGA modulated cytokine secretion, whose effects were targeted

204 towards IL-6 and IL-10, while having no impact on the other cytokines. In contrast, 4-HBA

205 and Pg-3-glc did not affect any of the cytokines tested. These results suggest that at least two 206 hydroxyl groups (either ortho or para to each other) might be required for bioactivity. In line

207 with this suggestion, potent radical scavenging activity of polyphenols was previously linked

208 to the presence of an ortho-dihydroxy group [22] and was furthermore correlated with the

209 number of hydroxyl groups on the B-ring [23]. In order to identify chemical structures or

210 properties required for anti-inflammatory effects, screening studies with a larger number of 211 related compounds are required.

\section{$212 \quad 2.5$ Conclusion}

213 In conclusion, the data suggest that PCA may possess anti-inflammatory properties through

214 modulation of IL-6 production, which could contribute to protective effects in inflammatory

215 diseases. Importantly, this action was observed at physiologically attainable concentrations,

216 although the effect was modest and limited to monocytes. The IL-6 and IL-10-reducing

217 effects of PGA were only observed at the higher, supraphysiological concentration and are

218 thus considered of limited physiological relevance. Pg-3-glc and 4-HBA had no effect on any

219 of the tested cytokines. Future studies should focus on screening a larger number of related

220 compounds in order to identify chemical structures or properties required for anti-

221 inflammatory effects and underlying mechanisms. 


\section{$222 \quad 2.6$ Acknowledgments}

223 The authors declare no conflicts of interest related to this experiment.

225 Funding: This work was supported by a BBSRC CASE studentship with GlaxoSmithKline

226 as industrial sponsor. The sponsors had no role in the interpretation of the study. The study 227 design was approved by GlaxoSmithKline. Apart from that, the sponsors had no role in the 228 collection, analysis and interpretation of the data, in preparation of the manuscript and in the 229 decision to publish. 
231 [1] Cassidy A, Mukamal KJ, Liu L, Franz M, Eliassen AH, Rimm EB. High anthocyanin intake is associated 232 with a reduced risk of myocardial infarction in young and middle-aged women. Circulation. 2013;127:188-96.

233 [2] Jennings A, Welch AA, Fairweather-Tait SJ, Kay C, Minihane A-M, Chowienczyk P, et al. Higher 234 anthocyanin intake is associated with lower arterial stiffness and central blood pressure in women. Am J Clin 235 Nutr. 2012;96:781-8.

236 [3] Noratto GD, Angel-Morales G, Talcott ST, Mertens-Talcott SU. Polyphenolics from acai (Euterpe oleracea Mart.) and red muscadine grape (Vitis rotundifolia) protect human umbilical vascular endothelial cells (HUVEC) from glucose- and lipopolysaccharide (LPS)-induced inflammation and target MicroRNA-126. J Agric Food Chem. 2011;59:7999-8012.

240 [4] Del Corno M, Varano B, Scazzocchino B, Filesi C, Masella R, Gessani S. Protocatechuic acid inhibits 241 human dentritic cell functional activation: Role of PPARy up-modulation. Immunobiology. 2014;219:416-24.

242 [5] Min SW, Ryu SN, Kim DH. Anti-inflammatory effects of black rice, cyanidin-3-O-beta-D-glycoside, and its metabolites, cyanidin and protocatechuic acid. Int Immunopharmacol. 2010;10:959-66.

[6] di Gesso JL, Kerr JS, Zhang QZ, Raheem S, Yalamanchili SK, O'Hagan D, et al. Flavonoid metabolites reduce tumor necrosis factor-a secretion to a greater extent than their precursor compounds in human THP-1 monocytes. Mol Nutr Food Res. 2015;59:1143-54.

[7] Amin HP, Czank C, Raheem S, Zhang QZ, Botting NP, Cassidy A, et al. Anthocyanins and their physiologically relevant metabolites alter the expression of IL-6 and VCAM-1 in CD40L and oxidized LDL challenged vascular endothelial cells. Mol Nutr Food Res. 2015;59:1095-106.

250 [8] Rodriguez-Mateos A, Heiss C, Borges G, Crozier A. Berry (poly)phenols and cardiovascular health. J Agric 251 Food Chem. 2014;62:3842-51.

252 [9] Erlund I, Koli R, Alfthan G, Marniemi J, Puukka P, Mustonen P, et al. Favorable effects of berry consumption on platelet function, blood pressure, and HDL cholesterol. Am J Clin Nutr. 2008;87:323-31.

[10] Ait-Oufella H, Taleb S, Mallat Z, Tedgui A. Recent advances on the role of cytokines in atherosclerosis. 255 Arterioscler Thromb Vasc Biol. 2011;31:969-79.

[11] Mullen W, Edwards CA, Serafini M, Crozier A. Bioavailability of pelargonidin-3-O-glucoside and its metabolites in humans following the ingestion of strawberries with and without cream. J Agric Food Chem. 2008;56:713-9.

259 [12] Sandhu AK, Huang YC, Xiao D, Park E, Edirisinghe I, Burton-Freeman B. Pharmacokinetic 260 characterization and bioavailability of strawberry anthocyanins relative to meal intake. J Agric Food Chem. $261 \quad 2016 ; 64: 4891-9$.

262 [13] Banaszewski K, Park E, Edirisinghe I, Cappozzo J, Burton-Freeman BM. A pilot study to investigate 263 bioavailability of strawberry anthocyanins and characterize postprandial plasma polyphenols absorption patterns 264 by Q-TOFLC/MS in humans. J Berry Res. 2013;3:113-26.

265 [14] Russell WR, Scobbie L, Labat A, Duthie GG. Selective bio-availability of phenolic acids from Scottish 266 strawberries. Mol Nutr Food Res. 2009;53:S85-S91.

267 [15] Azzini E, Vitaglione P, Intorre F, Napolitano A, Durazzo A, Foddai MS, et al. Bioavailability of strawberry 268 antioxidants in human subjects. Br J Nutr. 2010;104:1165-73.

269 [16] de Ferrars RM, Cassidy A, Curtis P, Kay CD. Phenolic metabolites of anthocyanins following a dietary 270 intervention study in post-menopausal women. Mol Nutr Food Res. 2014;58:490-502. 
271 [17] de Ferrars RM, Czank C, Zhang Q, Botting NP, Kroon PA, Cassidy A, et al. The pharmacokinetics of 272 anthocyanins and their metabolites in humans. Br J Pharmacol. 2014;171:3268-82.

273 [18] Tedgui A, Mallat Z. Cytokines in atherosclerosis: pathogenic and regulatory pathways. Physiol Rev. $274 \quad 2006 ; 86: 515-81$.

275 [19] Kavanagh IC, Symes CE, Renaudin P, Nova E, Mesa MD, Boukouvalas G, et al. Degree of oxidation of 276 low density lipoprotein affects expression of CD36 and PPAR gamma, but not cytokine production, by human 277 monocyte-macrophages. Atherosclerosis. 2003;168:271-82.

278 [20] Steensberg A, Fischer CP, Keller C, Moller K, Pedersen BK. IL-6 enhances plasma IL-1ra, IL-10, and 279 cortisol in humans. Am J Endocrinol Metab. 2003;285:E433-E7.

280 [21] Crouvezier S, Powell B, Keir D, Yaqoob P. The effects of phenolic components of tea on the production of 281 pro- and anti-inflammatory cytokines by human leukocytes in vitro. Cytokine. 2001;13:280-6.

282 [22] Yokozawa T, Chen CP, Dong E, Tanaka T, Nonaka GI, Nishioka I. Study on the inhibitory effect of tannins 283 and flavonoids against the 1,1-diphenyl-2-picrylhydrazyl radical. Biochem Pharmacol. 1998;56:213-22.

284 [23] Yi L, Chen CY, Jin X, Mi MT, Yu B, Chang H, et al. Structural requirements of anthocyanins in relation to 285 inhibition of endothelial injury induced by oxidized low-density lipoprotein and correlation with radical 286 scavenging activity. FEBS Lett. 2010;584:583-90. 